\title{
Phase I study of patients with non-muscle invasive bladder cancer (NMIBC) treated with vesigenurtacel-L (HS-410) after Bacillus Calmette-Guérin (BCG)
}

\author{
Gary Steinberg ${ }^{1}$, Neal Shore ${ }^{2}$, Lawrence Karsh ${ }^{3}$, James Bailen ${ }^{4}$, Michael Woods $^{5}$, Taylor Schreiber ${ }^{6}$, Melissa Price ${ }^{6 *}$ \\ From 30th Annual Meeting and Associated Programs of the Society for Immunotherapy of Cancer (SITC 2015) \\ National Harbor, MD, USA. 4-8 November 2015
}

\section{Background}

Vesigenurtacel-L (HS-410), consists of an allogeneic bladder cancer cell line, selected for high expression of a series of tumor antigens that are known to be shared by a high proportion of bladder tumors. The cell line secretes gp96-Ig, a modified heat shock protein which delivers cellderived antigens to MHC-I via the cross-presentation pathway, leading to preferential activation of CD8+ cytotoxic T cells. Vesigenurtacel-L was evaluated in a Phase I trial after treatment with standard of care induction BCG for safety and immune response.

\section{Methods}

Ten patients with non-muscle invasive bladder cancer who had undergone TURBT, were judged to be at an increased risk for recurrence, and were either BCG naïve or had completed previous BCG treatment $>12$ months prior to the most recent TURBT were treated with induction BCG as standard of care and enrolled in the trial. Patients received up to 15 doses of monotherapy vesigenurtacel-L at a dose of $10^{6}$ cells per dose, weekly for 12 weeks followed by 3 monthly doses. Baseline tumor tissue and any follow up biopsies were evaluated by immunohistochemistry for tumor infiltrating lymphocytes and expression of PD-L1.

\section{Results}

Vesigenurtacel-L was well tolerated with no treatmentrelated Grade 3/4 adverse events. The most common adverse events were low-grade injection site reactions consistent with delayed type hypersensitivity. Peripheral blood mononuclear cells were evaluated by flow cytometry for detection of circulating leukocyte subsets, regulatory $\mathrm{T}$ cells, myeloid derived suppressor cells, activated $\mathrm{T}$ cells and expression of immune checkpoint molecules on $\mathrm{T}$ cells. Additionally, analyses from pre- and posttreatment tissue specimens in a subset of patients including antigen expression, evaluation of tumor-infiltrating lymphocytes, PD-L1 expression and $\mathrm{T}$ cell receptor sequencing will be reported, and their extrapolation to an emerging definition of responder phenotype discussed. For example, tissue analysis indicates an increase in CD8+ $\mathrm{T}$ cells in the bladder after treatment, consistent with the mechanism of action derived from preclinical models, and this change appears be correlated to clinical outcome.

\section{Trial registration}

Clinical trial information: NCT02010203.

\begin{abstract}
Authors' details
${ }^{1}$ University of Chicago Medical Center, Chicago, IL, USA. ${ }^{2}$ Atlantic Urology Clinics, Myrtle Beach, SC, USA. ${ }^{3}$ The Urology Center of Colorado, Denver, CO, USA. ${ }^{4}$ First Urology, Jeffersonville, IN, USA. ${ }^{5}$ The University of North Carolina at Chapel Hill, Chapel Hill, NC, USA. ${ }^{6}$ Heat Biologics, Inc., Durham, NC, USA.
\end{abstract}

Published: 4 November 2015

doi:10.1186/2051-1426-3-S2-P447

Cite this article as: Steinberg et al:: Phase I study of patients with nonmuscle invasive bladder cancer (NMIBC) treated with vesigenurtacel-L (HS-410) after Bacillus Calmette-Guérin (BCG). Journal for ImmunoTherapy of Cancer 2015 3(Suppl 2):P447.

${ }^{6}$ Heat Biologics, Inc., Durham, NC, USA

Full list of author information is available at the end of the article 